\title{
Impact of hemodynamic goal-directed resuscitation on mortality in adult critically ill patients: a systematic review and meta- analysis
}

\author{
Maria Cronhjort $^{1,2}$ (D) Olof Wall $^{1} \cdot$ Erik Nyberg $^{2} \cdot$ Ruifeng Zeng $^{3} \cdot$ \\ Christer Svensen $^{1,2,4} \cdot$ Johan Mårtensson ${ }^{5,6}$ - Eva Joelsson-Alm ${ }^{1,2}$
}

Received: 20 February 2017 / Accepted: 29 May 2017 / Published online: 8 June 2017

(C) The Author(s) 2017. This article is an open access publication

\begin{abstract}
The effect of hemodynamic optimization in critically ill patients has been challenged in recent years. The aim of the meta-analysis was to evaluate if a protocolized intervention based on the result of hemodynamic monitoring reduces mortality in critically ill patients. We performed a systematic review and meta-analysis according to the Cochrane Handbook for Systematic Reviews of Interventions. The study was registered in the PROSPERO database (CRD42015019539). Randomized controlled trials published in English, reporting studies on adult patients treated in an intensive care unit, emergency department or equivalent level of care were included. Interventions had to be protocolized and based on results from hemodynamic measurements, defined as cardiac output, stroke volume,
\end{abstract}

Electronic supplementary material The online version of this article (doi:10.1007/s10877-017-0032-0) contains supplementary material, which is available to authorized users.

Maria Cronhjort

maria.cronhjort@sll.se

1 Department of Clinical Science and Education, Södersjukhuset, Karolinska Institutet, Stockholm, Sweden

2 Unit of Anaesthesiology and Intensive Care, Södersjukhuset, Stockholm, Sweden

3 The Second Hospital and Yuying Children's Hospital, Wenzhou Medical College, Wenzhou, China

4 Department of Anesthesiology, The University of Texas Medical Branch UTMB Health, John Sealy Hospital, Galveston, USA

5 Section of Anaesthesia and Intensive Care Medicine, Department of Physiology and Pharmacology, Karolinska Institutet, Solna, Sweden

6 Department of Intensive Care, Austin Hospital, Melbourne, VIC, Australia stroke volume variation, oxygen delivery, and central venous-or mixed venous oxygenation. The control group had to be treated without any structured intervention based on the parameters mentioned above, however, monitoring by central venous pressure measurements was allowed. Out of 998 screened papers, thirteen met the inclusion criteria. A total of 3323 patients were enrolled in the six trials with low risk of bias (ROB). The mortality was $22.4 \%$ (374/1671 patients) in the intervention group and 22.9\% (378/1652 patients) in the control group, OR 0.94 with a $95 \%$ CI of $0.73-1.22$. We found no statistically significant reduction in mortality from hemodynamic optimization using hemodynamic monitoring in combination with a structured algorithm. The number of high quality trials evaluating the effect of protocolized hemodynamic management directed towards a meaningful treatment goal in critically ill patients in comparison to standard of care treatment is too low to prove or exclude a reduction in mortality.

Keywords Critical care · Hemodynamic monitoring · Protocol · Fluid therapy · Meta-analysis · Mortality

\section{Introduction}

Hemodynamic optimization in critically ill patients is performed very differently in intensive care settings worldwide $[1,2]$. The obvious reason to give a patient fluid would be to improve cardiac output and organ perfusion. The challenge is to identify patients who might benefit from fluid, since only $50 \%$ of patients are considered to be fluid responders in a general intensive care population [3]. In a recent global observational study of fluid challenges in critically ill patients, only $22 \%$ of the patients were evaluated with dynamic indices for fluid 
responsiveness, such as Stroke Volume Variation (SVV), Pulse Pressure Variation (PPV), or a change in Stroke Volume (SV) after a Passive Leg Raising (PLR) test. Regardless of the response to the first fluid bolus approximately $50 \%$ of the patients received a second bolus of fluid [4]. Furthermore, only $8.2 \%$ of fluid boluses given to patients in septic shock in French ICUs were monitored with continuous measurements of cardiac output [5]. To assess fluid responsiveness many clinicians use clinical examination, pulse rate, blood pressure, central venous pressure (CVP) or urinary production. However, such static measures are poor markers of fluid responsiveness [6-9]. Logically, the use of dynamic parameters such as real-time changes in SV or cardiac output should provide more accurate measures of the physiological response to fluid therapy. Since monitoring alone cannot be expected to improve outcome, it is important to combine the monitoring with a structured intervention.

Goal-directed fluid therapy (GDT) is widely used in the perioperative setting and has been shown to reduce mortality [10] and surgical complications in small trials [11-13]. However, this concept was recently challenged in a large randomized controlled trial [14], showing no reduction in mortality or morbidity. Yet, a subsequent meta-analysis demonstrated that GDT was associated with fewer infections and shorter duration of hospital stay. Another recent meta-analysis showed that GDT based on dynamic parameters reduced morbidity in the perioperative setting [15].

Critically ill patients react differently to fluids compared to patients who present for elective surgery. For patients with sepsis the problem of vasodilatation and increased capillary permeability reduces the peak effect and duration of the volume expanding effect of intravenous fluids. The concept of Early Goal-Directed Therapy (EGDT) was introduced by Rivers et al. 2001 as a specific treatment protocol utilized for the first $6 \mathrm{~h}$ in patients with severe sepsis or septic shock [16]. However, recently three large clinical trials randomizing $>4000$ septic patients to early goal directed therapy (EGDT) did not show any reduction in mortality from the protocolized approach [17-19]. Angus et al. performed a meta-analysis of the effect of EGDT in sepsis [20], where there was no reduction in mortality in the EGDT-groups. Furthermore, there is a need to evaluate the effect of hemodynamic optimization in all critically ill patients in the ICU and in patients where treatment algorithms have been used longer than $6 \mathrm{~h}$. The aim of this meta-analysis was to evaluate if hemodynamic monitoring combined with a structured and extended treatment plan before or during the stay in the ICU reduces mortality in critically ill patients.

\section{Materials and methods}

We performed a systematic review and meta-analysis according to the Cochrane Handbook for Systematic Reviews of Interventions [21]. The Preferred Reporting Items for Systematic Review and Meta-analysis Protocols (PRISMA-P) statement was followed in setting up and reporting the meta-analysis [22]. The quality of evidence was assessed using the GRADE system [23]. The study was registered in the PROSPERO database (CRD42015019539).

\subsection{Type of studies}

We only included randomized clinical trials (RCT) published in English.

\subsection{Inclusion criteria}

To define the meta-analysis, we used the Cochrane acronym PICO (Participants, Interventions, Comparators, and Outcomes).

\subsubsection{Participants}

Participants were adult patients treated at an ICU, emergency department or corresponding level of care.

\subsubsection{Intervention}

Interventions had to be protocolized and based on results from hemodynamic measurements, defined as cardiac output (CO), SV, SVV, oxygen delivery, central venous oxygenation $(\mathrm{ScvO} 2)$ or mixed venous oxygenation (SvO2).

\subsubsection{Comparators}

The control group had to be treated with standard of care, without any structured intervention based on the parameters mentioned above, however, monitoring by CVP measurements were allowed.

\subsubsection{Outcome}

Primary outcome was all-cause mortality at any time point. Positive fluid balance and weight gain were defined as secondary endpoints. 


\subsection{Exclusion criteria}

Exclusion criteria were perioperative, pediatric and animal studies. If the intervention was initiated before or in the operating room, the intervention was defined as perioperative.

\subsection{Search strategy}

We searched the Pub Med, Embase and CENTRAL databases for articles using the following search terms: [("intensive care" OR "intensive care units" OR "ICU" OR critically ill OR critical illness OR emergency service OR emergency department) AND (cardiovascular agents OR fluid therapy)]. The search was performed 18/12/2014 with an updated search 04/01/2016. The search strategy was adapted until all known articles were included in the search. Full search strategies are available in the Supplementary Appendix 1.

\subsection{Selection of studies}

The searches were performed with the assistance of a librarian and all titles and abstracts were first independently reviewed for relevancy by two persons in the team of reviewers (MC, EJA, OW, EN, and RZ). In addition, references lists and expert opinions were reviewed for further relevant studies. Full-texts of articles that could meet inclusion criteria were read by two reviewers and either included or excluded. If there were different opinions two more reviewers examined the article and consensus was reached after a joint discussion in the team.

\subsection{Data extraction}

Two reviewers per article used a data collection form customized from the standardized Cochrane Collaborative form to extract data from each article. All relevant information such as study size, type of monitoring, intervention and control used, primary and secondary outcomes were registered using this form.

\subsection{Quality assessment}

Risk of bias (ROB) was assessed independently by two reviewers using the Cochrane Collaborative Tool for Risk of Bias Assessment [21]. It is not possible to blind the treating staff from what hemodynamic algorithm is being used. Thus we moderated the intention in the pre-specified plan (PROSPERO CRD42015019539) to disregard non-blinding in the ROB assessment.

\subsection{Statistical methods}

Outcome measurement was expressed as pooled OR with a $95 \%$ CI, presented as a forest plot. We used the $\chi^{2}$ statistic to assess statistical heterogeneity of treatment effect, where a p-value $<0.10$ was interpreted as evidence of heterogeneity. We also used the $\mathrm{I}^{2}$ statistic to assess the impact of statistical heterogeneity on the treatment effect. $\mathrm{I}^{2} 0-40 \%$ was interpreted as if the inconsistency might not be important. We used the Mantel-Haenszel random effects model even if significant statistical heterogeneity was not present This was because the assumptions of the fixed effect model were not fulfilled (the same direction and size of effect in all studies). We also had large clinical heterogeneity. $\tau^{2}$ was used to describe the estimate of between study variance in the random effects analyses. Sensitivity analyses were performed for arbitrary decisions; with and without trials with more than one domain with unclear or high risk of bias and with/without a validated hemodynamic measurement tool. We performed a Trial Sequential Analysis (TSA) to calculate the required sample size to be able to exclude a random finding of no effect $[24,25]$. The Trial Sequential Software (Copenhagen Trial Unit, Copenhagen, Denmark) was used for the TSA [26]. We considered results as statistically significant with a two-sided $p$-value $<0.05$. The statistical analyses were performed by RevMan 5.3 software, The Nordic Cochrane Centre, Copenhagen, Denmark.

\section{Results}

Out of 998 screened papers, thirteen met the inclusion criteria (Fig. 1). A total of 6850 patients were enrolled in the included studies. There were 3323 patients in the six trials with low ROB. No studies reported the secondary endpoints of positive fluid balance or body weight.

\subsection{Study characteristics}

The trials that were included and their characteristics are described in Table 1 [16-19, 27-35]. All were randomized clinical open-label trials. Five were EGDT trials [16-19, 31] which recruited 4735 patients and eight trials were not EGDT protocol studies which recruited 2115 patients. Six trials were assessed as overall low ROB, five studies were unclear or high risk in one other domain, and two studies were unclear or high in two or more other domains (Fig. 2). Low inclusion to screening rate without explanations lead to the exclusion of two recent large multicentre EGDT 

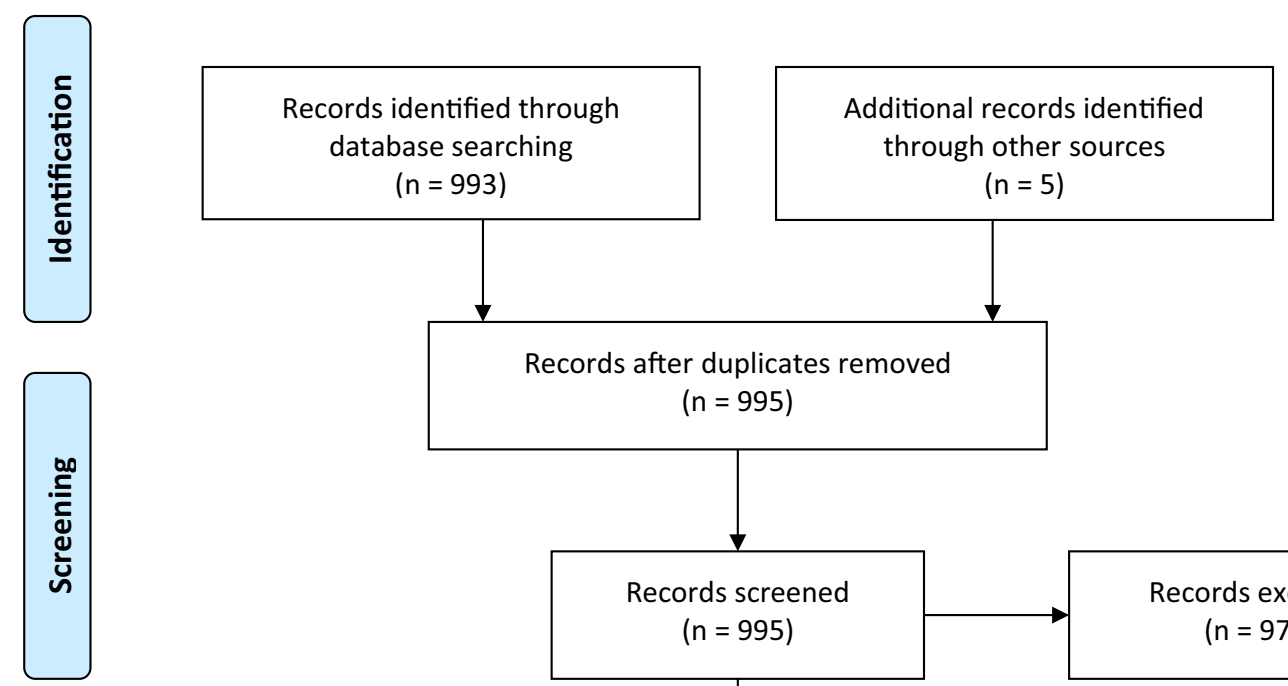

Records after duplicates removed $(n=995)$
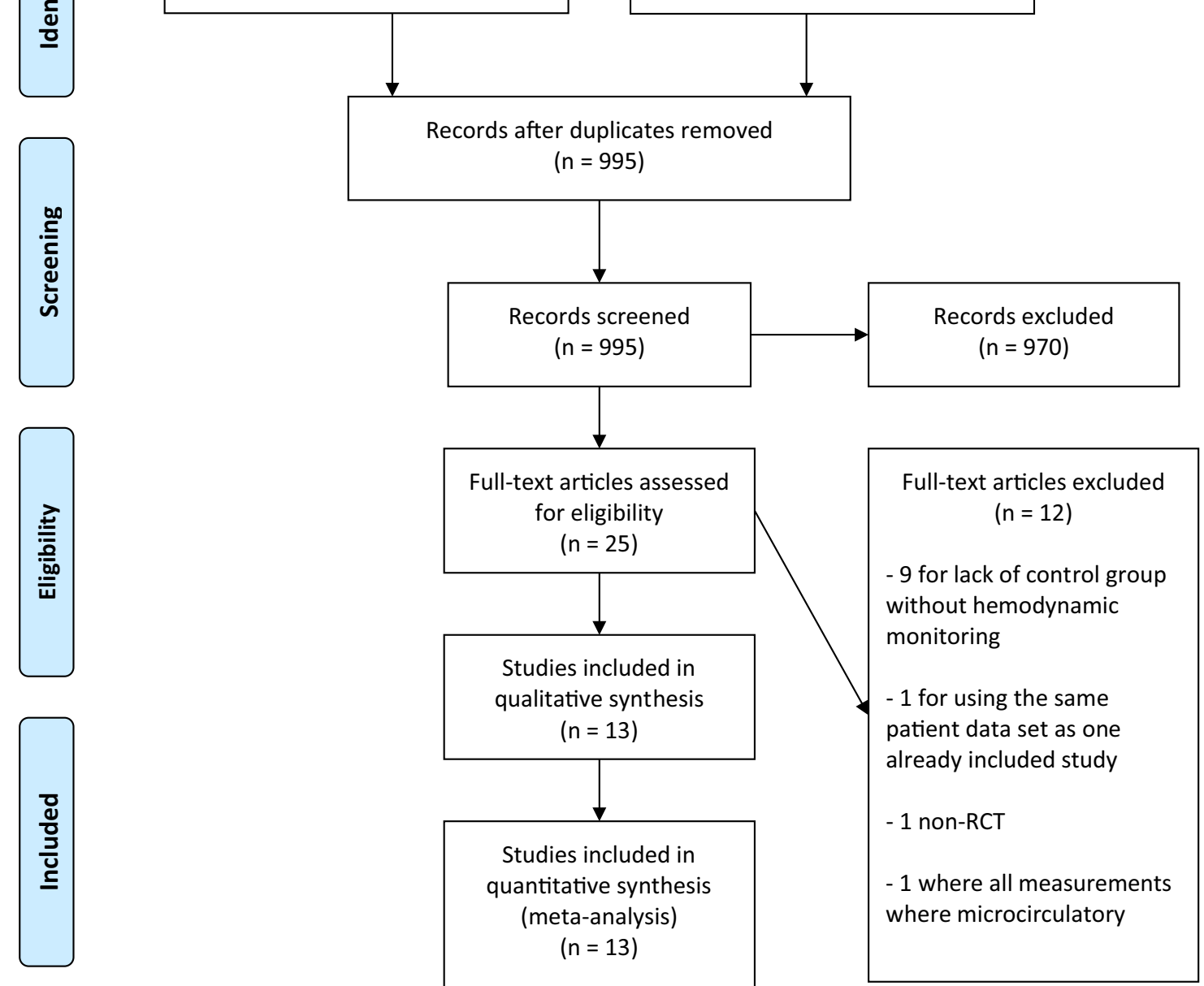

Fig. 1 PRISMA flow diagram Fig. 1 Legend: PRISMA flow diagram on literature search and study selection

trials [17, 19]. Detailed ROB assessments are available in Supplementary Appendix 2.

The analysis including the six trials with low risk of bias $[16,18,27,28,31,33]$ resulted in a mortality rate of $22.4 \%(374 / 1671$ patients) in the intervention group and $22.9 \%(378 / 1652$ patients) in the control group, OR 0.94 with a $95 \%$ CI of $0.73-1.22$. Exploring the heterogeneity of treatment effect in the included studies with the $\chi^{2}$ statistic showed no evidence of heterogeneity, but according to the $\mathrm{I}^{2}$ statistic the percentage of variability of the effect estimate that was due to heterogeneity was large; $\mathrm{I}^{2}=41 \%$ (Fig. 3).

In the analysis of all the thirteen included trials the mortality was $23.8 \%(766 / 3222$ patients) in the intervention group versus $23.5 \%$ (851/3628 patients) in the control group with an OR of 1.00 (95\% CI 0.89-1.12), see Fig. 4. There was no statistically significant difference in mortality between the groups regardless of whether just the trials with low ROB were analyzed or all trials. However, the TSA showed that a sample size of 17,532 patients would 


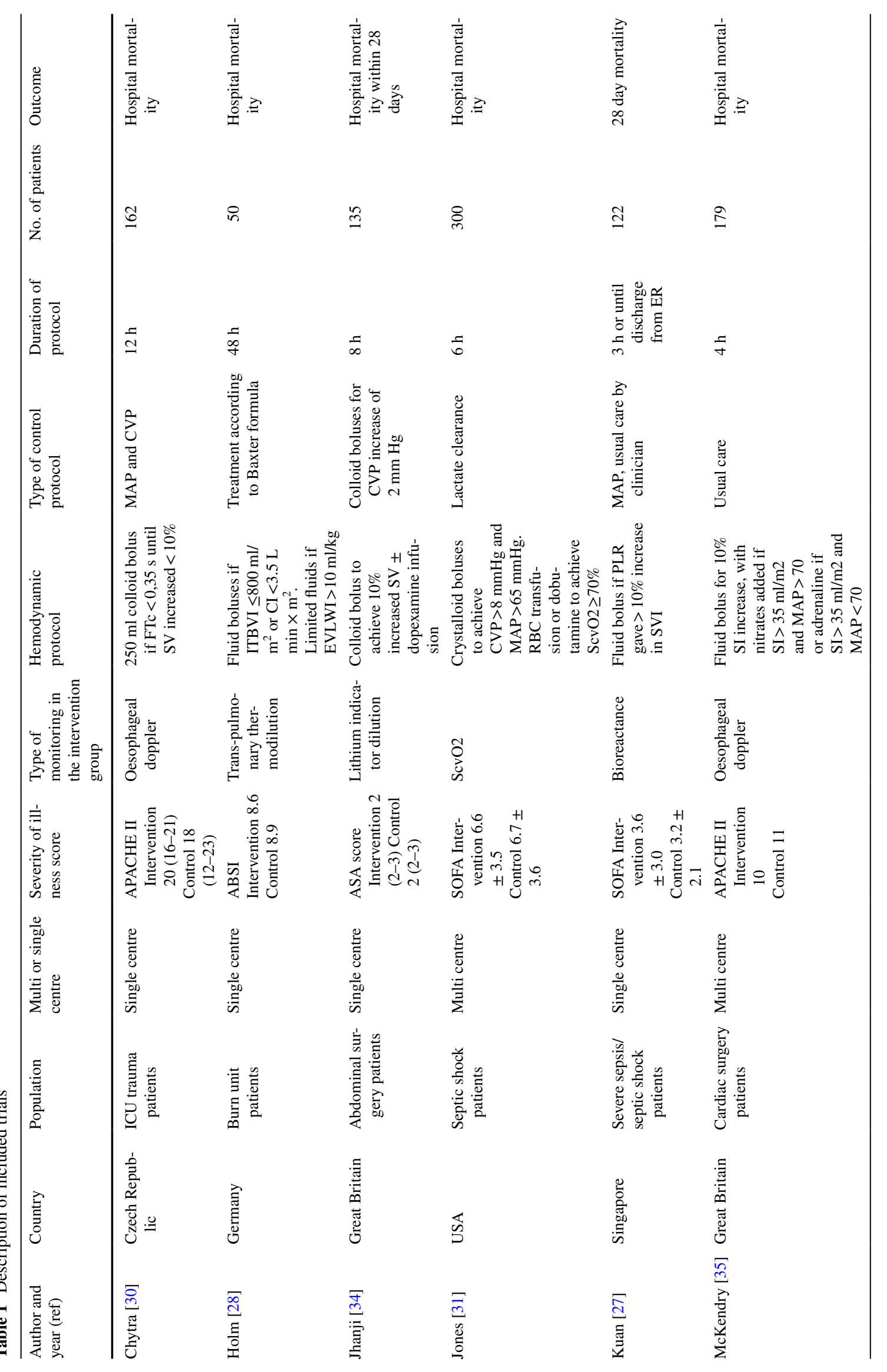




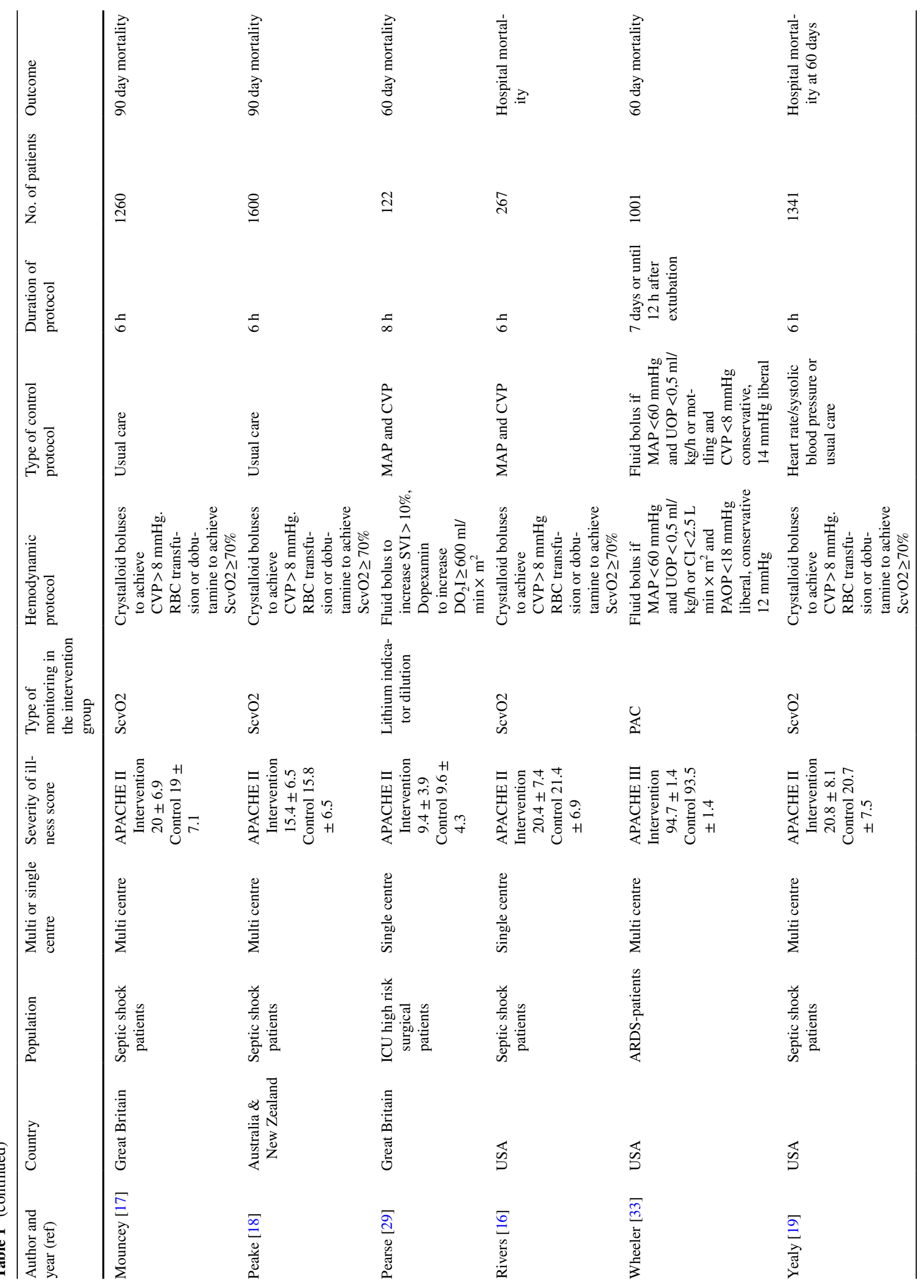




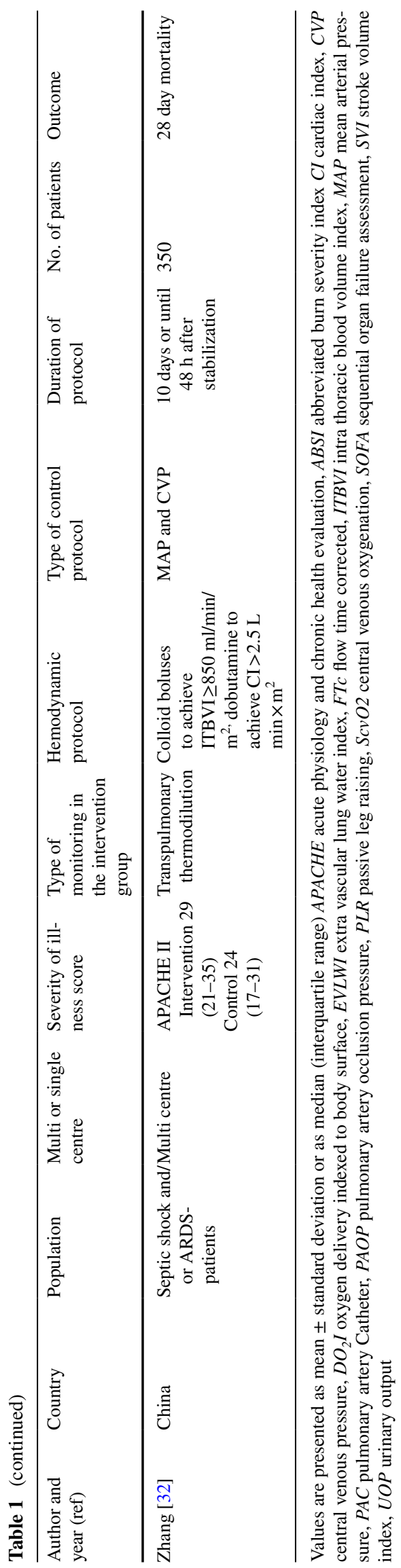

have been needed to be able to exclude that the negative finding was random.

\subsection{Results of sensitivity analyses}

Analysis of the ten trials with a validated hemodynamic measurement technique, excluding the trials that used bioreactance [27] and esophageal doppler [30, 35], resulted in a mortality of $24.8 \%$ (743/2992 patients) in the intervention group and $24.3 \%$ (825/3400 patients) in the controls, with an OR of $1.01,95 \%$ CI $0.89-1.13$.

The quality of the evidence according to GRADE was considered as moderate $(\oplus \oplus \oplus \Theta)$.

\section{Discussion}

We found no statistically significant reduction in mortality from hemodynamic optimization using hemodynamic monitoring in combination with a structured algorithm in the analysis of the six trials with low ROB or in the analysis with all thirteen trials. The sample size of the metaanalysis was too small to exclude that the negative effect was random. There is a paucity of studies looking specifically at hemodynamically guided interventions in the critically ill. Mortality was low in the control group compared to many sepsis trials, which might depend on the mix of patients (including trauma, burn patients and high risk surgical patients as well as septic shock patients) and the time at which mortality was measured (hospital/28 days $/ 90$ days). The effect of an intervention on mortality would be expected to be smaller if the mortality in the control group is low. In contrast to our result, there are several meta-analyses that found positive effects of hemodynamic optimization $[36,37]$ but they mainly included studies that were investigations of mortality before and after introduction of an EGDT protocol. A recent meta-analysis of RCTs by $\mathrm{Gu}$ et al. concluded that there is a positive effect of EGDT [38]. They stated that the control group received standard therapy or usual care. However, there were violations of the study protocol since they have included five trials where the control groups were treated according to hemodynamic protocols [39-43]. Moreover, they included trials with high risk of bias in the analysis. Finally, their meta-analysis was conducted before the publication of the Australasian Resuscitation In Sepsis Evaluation (ARISE) [44] and the Protocolized Management in Sepsis (PROMISE) [17] trials which contributed with a substantial number of patients. Our results are consistent with a more recent meta-analysis by Angus et al. limited to septic patients [20]. They included studies that compared EGDT to other hemodynamic protocols or standard of care. They included 13 trials; the same five EGDT trials as in our meta-analysis plus one pediatric 


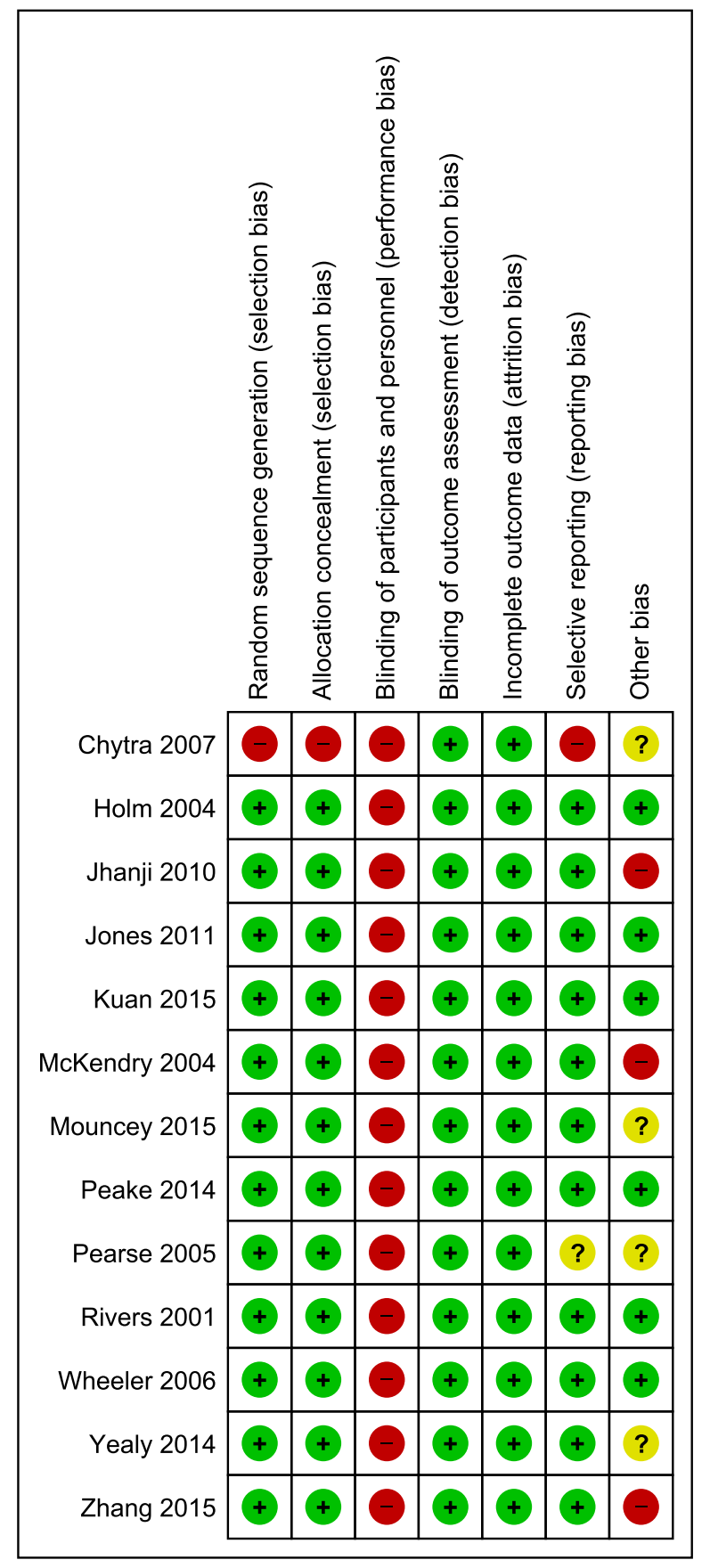

Fig. 2 Risk of bias assessment for included studies Fig. 2 Legend: Assessment of validity of included studies according to the cochrane collaborative tool for risk of bias assessment. Low risk of bias + , high risk of bias -, unclear risk of bias?

[45] and five articles published in Chinese [46-50]. They found no reduction in mortality by EGDT; mortality was $23.2 \%$ in the EGDT group compared to $22.4 \%$ in the control group, OR 1.01 (95\% CI 0.88-1.16). They found only 2 out of 13 studies with low risk of bias, but they included all trials in the analysis. Poeze et al. performed a metaanalysis 2005 , using the same hemodynamic variables as in our meta-analysis [10]. They also included studies where two hemodynamic protocols were compared. Consequently, the only overlapping study with our meta-analysis was the Rivers trial [16]. They found no reduction in mortality in patients with septic shock or organ failure.

Our results add to the current knowledge by investigating a broader population cohort without limiting the selection to patients with sepsis. We only included studies with a structured intervention where the controls were treated according to usual care or with simple hemodynamic protocols based on CVP, MAP or lactate or a combination of those parameters. We performed the meta-analysis according to the Cochrane handbook for systematic reviews of interventions and excluded studies with high ROB with the exception of the domain of blinding. Our results stress the lack of studies that evaluate relevant goals for hemodynamic optimization of critically ill patients.

A key question while performing a meta-analysis is the quality of the included studies. Even though we used the structured method of bias assessment according to Cochrane, we found that there were other weaknesses in the trials that were not detected by this method. To investigate if mortality can be reduced by individualized hemodynamic management the monitoring technique should be accurate and precise. During the last decade, several less invasive techniques to measure cardiac output have been introduced [51]. Unfortunately, there are no generally accepted criteria for acceptable agreement that can be applied in the validation of cardiac output measurement techniques. There are criteria suggested by Critchley and Critchley [52], but these are not always followed [53]. The main issue is that there is a constant variation of cardiac output in humans, thus no real precision of measurement can be obtained, At best, estimation of the precision is actually just serial measurements [54]. However, nine of the trials used measurement techniques that are generally accepted as validated in critically ill patients. A sensitivity analysis with these trials showed slightly higher mortality in the intervention group than in the controls, a non-significant result. This neither proved or refuted the hypothesis that it is important to use a validated measurement technique, maybe because the effect of the intervention was small. One trial used a PAC [55], which is considered the gold standard of clinical cardiac output measurement. Two studies used transpulmonary thermodilution $[28,32]$ which has been validated against PAC and been found to be sufficiently accurate for clinical purposes [56]. Two studies used a lithium dilution technique [29, 34], which is another validated dilution technique [57]. Five trials used continuous $\mathrm{ScvO} 2$ to estimate the need for fluids, inotropic agents and blood transfusions according to the EGDT protocol. This method has been validated against intermittent $\mathrm{ScvO} 2$ measurements with blood gas analyzers and been found to have excellent 


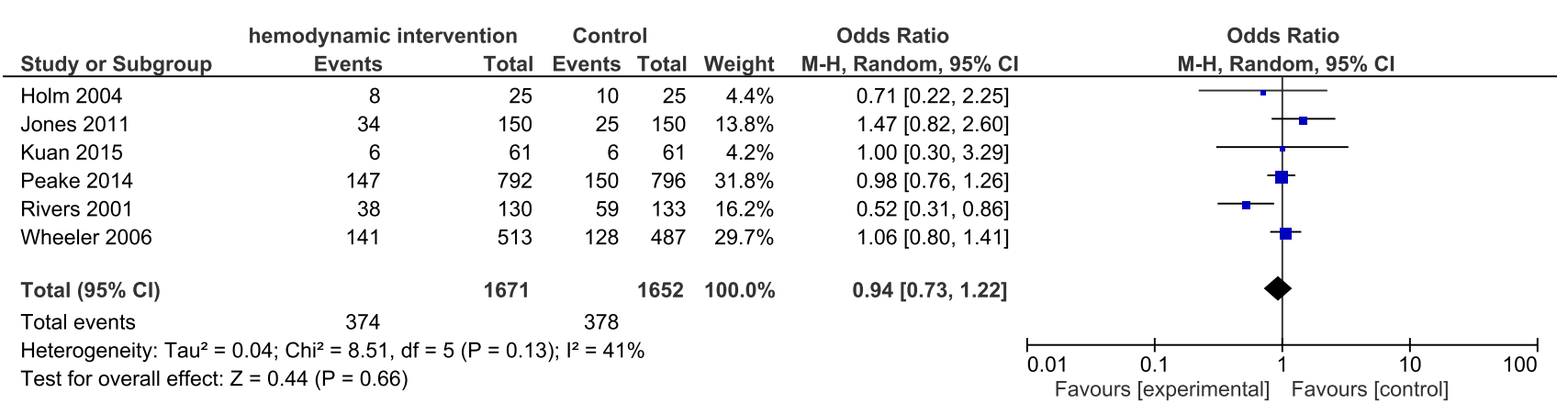

Fig. 3 Meta-analysis of effectiveness of hemodynamic monitoring combined with protocolized interventions to reduce mortality, low risk of bias trials Fig. 3 Legend: Meta-analysis of effectiveness of hemodynamic monitoring combined with protocolized interventions to reduce mortality, low risk of bias trials. Weight is the relative contribution of each study to the overall treatment effect (odds risk ratio and $95 \%$ confidence interval) on a log scale assuming Mantel-Haenszel random effects model

\begin{tabular}{|c|c|c|c|c|c|}
\hline \multirow[b]{2}{*}{ Study or Subgroup } & \multicolumn{2}{|c|}{ hemodynamic intervention } & \multicolumn{2}{|c|}{ Control } & \multirow[b]{2}{*}{ Weight } \\
\hline & Events & Total & Events & Total & \\
\hline McKendry 2004 & 4 & 89 & 2 & 85 & $0.4 \%$ \\
\hline Kuan 2015 & 6 & 61 & 6 & 61 & $0.9 \%$ \\
\hline Holm 2004 & 8 & 25 & 10 & 25 & $1.0 \%$ \\
\hline Jhanji 2010 & 9 & 90 & 6 & 45 & $1.1 \%$ \\
\hline Pearse 2005 & 7 & 62 & 9 & 60 & $1.2 \%$ \\
\hline Chytra 2007 & 13 & 80 & 18 & 82 & $2.1 \%$ \\
\hline Jones 2011 & 34 & 150 & 25 & 150 & $4.0 \%$ \\
\hline Rivers 2001 & 38 & 130 & 59 & 133 & $5.1 \%$ \\
\hline Zhang 2015 & 83 & 168 & 90 & 182 & $7.6 \%$ \\
\hline Yealy 2014 & 92 & 439 & 167 & 902 & $16.4 \%$ \\
\hline Wheeler 2006 & 141 & 513 & 128 & 487 & $17.0 \%$ \\
\hline Peake 2014 & 147 & 792 & 150 & 796 & $20.9 \%$ \\
\hline Mouncey 2015 & 184 & 623 & 181 & 620 & $22.3 \%$ \\
\hline Total $(95 \% \mathrm{Cl})$ & & 3222 & & 3628 & $100.0 \%$ \\
\hline Total events & 766 & & 851 & & \\
\hline \multicolumn{6}{|c|}{ Heterogeneity: $\mathrm{Tau}^{2}=0.00 ; \mathrm{Chi}^{2}=11.91, \mathrm{df}=12(\mathrm{P}=0.45) ; \mathrm{I}^{2}=0 \%$} \\
\hline
\end{tabular}

Fig. 4 Meta-analysis of effectiveness of hemodynamic monitoring combined with protocolized interventions to reduce mortality, all included trials Fig. 4 Legend: Meta-analysis of effectiveness of hemodynamic monitoring combined with protocolized interventions

accuracy and acceptable precision [58]. Two trials used measurement techniques which are not as well validated in critically ill patients. One of those trials used thoracic bioreactance [27], where there are conflicting results from validation. A meta-analysis has shown that precision is too poor for clinical use [59] while others deemed the accuracy and precision acceptable for clinical use in most situations [60]. Esophageal doppler was used in two trials [30, 35]. It is a technique where measurements depend entirely on correct positioning of the probe which can be cumbersome in the critical care setting. It is mainly used perioperatively, whereas validation in the intensive care setting has shown significant underestimation of $\mathrm{CO}$, especially in women [61].

For a hemodynamic algorithm to be able to reduce mortality the intervention must be directed towards a
Odds Ratio Odds Ratio

M-H, Random, $95 \% \mathrm{Cl}$

$1.95[0.35,10.95]$

$1.00[0.30,3.29]$

$0.71[0.22,2.25]$

$0.72[0.24,2.17]$

$0.72[0.25,2.08]$

$0.69[0.31,1.52]$

$1.47[0.82,2.60]$

$0.52[0.31,0.86]$

$1.00[0.66,1.52]$

$1.17[0.88,1.55]$

$1.06[0.80,1.41]$

$0.98[0.76,1.26]$

$1.02[0.80,1.30]$

$1.00[0.89,1.12]$ M-H, Random, $95 \% \mathrm{CI}$

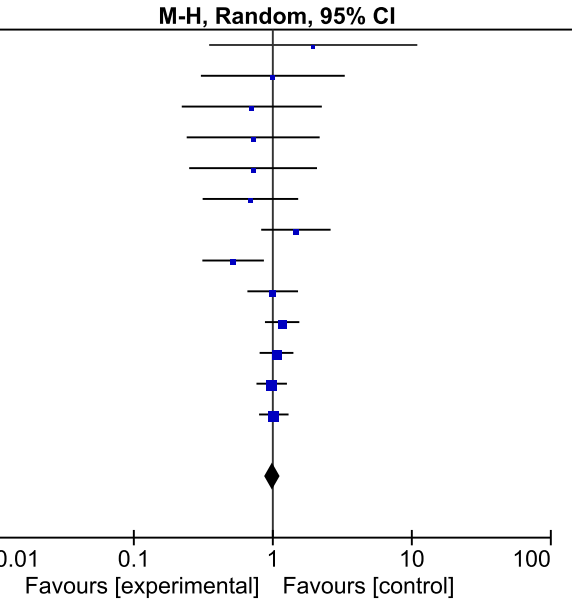

to reduce mortality, all included trials. Weight is the relative contribution of each study to the overall treatment effect (odds risk ratio and 95\% confidence interval) on a log scale assuming Mantel-Haenszel random effects model

meaningful treatment goal. Dynamic parameters have been shown to reflect fluid responsiveness better than static parameters and the use of dynamic parameters are recommended over static parameters by experts in the European Society of Intensive Care [62]. Only one of the included trials used a dynamic parameter; the difference in stroke volume index (SVI) caused by a PLR test [27]. This was a small trial using a less precise CO-measurement (bioreactance) and not dimensioned to show a mortality difference. The three large trials that replicated the original EGDT protocol by Rivers did not show any mortality benefit from the protocol using $\mathrm{ScvO} 2$ compared to standard of care. A problem with $\mathrm{ScvO} 2$ is that most patients in septic shock would have a hyperdynamic circulation with supranormal $\mathrm{ScvO} 2$ values, hence the hemodynamic protocol does not lead to a change of treatment. In a small Dutch study 
looking at $\mathrm{ScvO} 2$ values in patients with septic shock the mean $\mathrm{ScvO} 2$ was $74 \% \pm 10$. Only $6 \%$ had a $\mathrm{ScvO} 2<60 \%$ [63]. $\mathrm{ScvO} 2$ is used as a surrogate measurement for oxygen saturation in mixed venous blood $(\mathrm{SvO} 2)$, and like $\mathrm{SvO} 2$ it depends on $\mathrm{Hb}$, arterial saturation and $\mathrm{CO}$ as well as oxygen demand. There is great inter patient variability in $\mathrm{ScvO} 2$, since it reflects the attempts of the organs to extract the demanded oxygen [64]. The EGDT trials also used CVP to guide the initial fluid therapy, which we excluded from this meta-analysis because it has been repeatedly proven that CVP is of limited value in predicting fluid responsiveness [9]. In a recent meta-analysis with individual patient data a low CVP of $2-8 \mathrm{mmHg}$ had a positive predictive value for fluid responsiveness from 56 to $65 \%$ [6].

To answer the question if a critically ill patient ought to be hemodynamically monitored the controls must receive standard care without hemodynamic monitoring. In this meta-analysis we chose to disregard CVP as a hemodynamic monitoring tool, due to the poor correlation with fluid responsiveness.

There may be several ways of understanding the lack of evidence of benefit from individualized hemodynamic management although it has been a key feature of intensive care ever since the introduction of the PAC [65]. Either the task of designing and performing a clinical trial with an algorithm that will suit all critically ill patients is too challenging. Or maybe clinical judgment based on many different parameters as temperature and color of the skin, mental impairment, urinary output and blood pressure is a composite variable that seldom can be reflected by a single hemodynamic measurement. Perhaps we have still to find relevant goals for hemodynamic optimization of critically ill patients. Another explanation might be the heterogeneity in critically ill patients. It is possible that only a subgroup of the most severely ill patients might benefit from structured hemodynamic optimization. That hemodynamic optimization is unnecessary could also be a possible conclusion, but there are not enough studies to prove this.

Our meta-analysis has several strengths. It was planned according to the PRISMA guidelines, registered at PROSPERO and performed according to the Cochrane methodology. A broad search strategy was used and a broad spectrum of studies was included, not only large EGDT trials. We further applied the clinical fact that most critically ill patients are not treated by advanced hemodynamic protocols [5] and investigated the results of studies where the controls were treated according to usual care or with simple hemodynamic protocols based on CVP, MAP or lactate or a combination of those parameters.

Limitations of our study are the clinical diversity in patients, protocols and hemodynamic measurements. The fact that different trials had different timeframes to look at mortality as endpoints is another limitation, as well as that
non-English language studies were not included. We did not include observational studies which limits the capacity to evaluate side effects of protocolized hemodynamic management. We also excluded trials that did not evaluate mortality, fluid balance or weight gain.

\section{Conclusions}

This meta-analysis contributes to new knowledge about hemodynamic monitoring combined with structured treatment of critically ill patients in the ICU. In contrast to recent meta-analyses, which only dealt with septic patients, this study also includes studies with other types of critically ill patients. Furthermore, we only included studies in which the intervention group was treated according to a structured plan while the control group was treated according to usual care or with simple hemodynamic protocols based on CVP, MAP or lactate or a combination of those parameters. There are too few high quality trials evaluating the effect of protocolized hemodynamic management directed towards a meaningful treatment goal in critically ill patients in comparison to standard of care treatment to prove or exclude a reduction in mortality.

Acknowledgements We thank librarian Christina Lindberg for her expert assistance in the literature searches.

Funding The study was supported by a research grant from Stockholm County Council. The funders had no role in study design, data collection or analysis, decision to publish, or preparation of the manuscript.

\section{Compliance with ethical standards}

Conflict of interest On behalf of all authors, the corresponding author states that there are no competing interests.

Research involving human participants No ethical permission was sought as the study did not involve human participants.

Open Access This article is distributed under the terms of the Creative Commons Attribution 4.0 International License (http:// creativecommons.org/licenses/by/4.0/), which permits unrestricted use, distribution, and reproduction in any medium, provided you give appropriate credit to the original author(s) and the source, provide a link to the Creative Commons license, and indicate if changes were made.

\section{References}

1. Cecconi M, Hofer C, Teboul JL, Pettila V, Wilkman E, Molnar Z, Della Rocca G, Aldecoa C, Artigas A, Jog S, Sander M, Spies C, Lefrant JY, De Backer D. Fluid challenges in intensive care: the FENICE study: a global inception cohort study. Intensive Care Med. 2015;41:1529-37. 
2. Glassford NJ, Martensson J, Eastwood GM, Jones SL, Tanaka A, Wilkman E, Bailey M, Bellomo R. Defining the characteristics and expectations of fluid bolus therapy: a worldwide perspective. J Crit Care. 2016;35:126-32.

3. Marik PE, Monnet X, Teboul JL. Hemodynamic parameters to guide fluid therapy. Ann Intensive Care. 2011;1:1.

4. Cecconi M, Hofer C, Teboul JL, Pettila V, Wilkman E, Molnar Z, Della Rocca G, Aldecoa C, Artigas A, Jog S, Sander M, Spies C, Lefrant JY, De Backer D, Investigators F, Group ET. Fluid challenges in intensive care: the FENICE study: a global inception cohort study. Intens Care Med 2015;41:1529-37.

5. Boulain T, Boisrame-Helms J, Ehrmann S, Lascarrou JB, Bougle A, Chiche A, Lakhal K, Gaudry S, Perbet S, Desachy A, Cabasson S, Geneau I, Courouble P, Clavieras N, Massanet PL, Bellec F, Falquet Y, Reminiac F, Vignon P, Dequin PF, Meziani F. Volume expansion in the first 4 days of shock: a prospective multicentre study in 19 French intensive care units. Intensive Care Med. 2015;41:248-56.

6. Eskesen TG, Wetterslev M, Perner A. Systematic review including re-analyses of 1148 individual data sets of central venous pressure as a predictor of fluid responsiveness. Intensive Care Med. 2016;42:324-32.

7. McGee S, Abernethy WB 3rd, Simel DL. The rational clinical examination. Is this patient hypovolemic? JAMA. 1999;281:1022-9.

8. Pierrakos C, Velissaris D, Scolletta S, Heenen S, De Backer D, Vincent JL. Can changes in arterial pressure be used to detect changes in cardiac index during fluid challenge in patients with septic shock? Intensive Care Med. 2012;38:422-8.

9. Marik PE, Baram M, Vahid B. Does central venous pressure predict fluid responsiveness? A systematic review of the literature and the tale of seven mares. Chest. 2008;134:172-8.

10. Poeze M, Solberg BC, Greve JW, Ramsay G. Monitoring global volume-related hemodynamic or regional variables after initial resuscitation: What is a better predictor of outcome in critically ill septic patients? Crit Care Med. 2005;33:2494-500.

11. Wakeling HG, McFall MR, Jenkins CS, Woods WG, Miles WF, Barclay GR, Fleming SC. Intraoperative oesophageal Doppler guided fluid management shortens postoperative hospital stay after major bowel surgery. Br J Anaesth. 2005;95:634-42.

12. Goepfert MS, Richter HP, Eulenburg CZ, Gruetzmacher J, Rafflenbeul E, Roeher K, von Sandersleben A, Diedrichs S, Reichenspurner H, Goetz AE, Reuter DA. Individually optimized hemodynamic therapy reduces complications and length of stay in the intensive care unit a prospective, randomized controlled trial. Anesthesiology. 2013;119:824-36.

13. Salzwedel C, Puig J, Carstens A, Bein B, Molnar Z, Kiss K, Hussain A, Belda J, Kirov MY, Sakka SG, Reuter DA. Perioperative goal-directed hemodynamic therapy based on radial arterial pulse pressure variation and continuous cardiac index trending reduces postoperative complications after major abdominal surgery: a multi-center, prospective, randomized study. Crit Care. 2013;17:R191.

14. Pearse RM, Harrison DA, MacDonald N, Gillies MA, Blunt M, Ackland G, Grocott MP, Ahern A, Griggs K, Scott R, Hinds C, Rowan K. Effect of a perioperative, cardiac output-guided hemodynamic therapy algorithm on outcomes following major gastrointestinal surgery: a randomized clinical trial and systematic review. JAMA. 2014;311:2181-90.

15. Benes J, Giglio M, Brienza N, Michard F. The effects of goaldirected fluid therapy based on dynamic parameters on post-surgical outcome: a meta-analysis of randomized controlled trials. Crit Care. 2014;18:584.

16. Rivers E, Nguyen B, Havstad S, Ressler J, Muzzin A, Knoblich B, Peterson E, Tomlanovich M. Early goal-directed therapy in the treatment of severe sepsis and septic shock. N Engl J Med. 2001;345:1368-77.

17. Mouncey PR, Osborn TM, Power GS, Harrison DA, Sadique MZ, Grieve RD, Jahan R, Harvey SE, Bell D, Bion JF, Coats TJ, Singer M, Young JD, Rowan KM. Trial of early, goal-directed resuscitation for septic shock. N Engl J Med. 2015;372:1301-11.

18. Peake SL, Delaney A, Bailey M, Bellomo R, Cameron PA, Cooper DJ, Higgins AM, Holdgate A, Howe BD, Webb SA, Williams P. Goal-directed resuscitation for patients with early septic shock. N Engl J Med. 2014;371:1496-506.

19. Yealy DM, Kellum JA, Huang DT, Barnato AE, Weissfeld LA, Pike F, Terndrup T, Wang HE, Hou PC, LoVecchio F, Filbin MR, Shapiro NI, Angus DC. A randomized trial of protocol-based care for early septic shock. N Engl J Med. 2014;370:1683-93.

20. Angus DC, Barnato AE, Bell D, Bellomo R, Chong CR, Coats TJ, Davies A, Delaney A, Harrison DA, Holdgate A, Howe B, Huang DT, Iwashyna T, Kellum JA, Peake SL, Pike F, Reade MC, Rowan KM, Singer M, Webb SA, Weissfeld LA, Yealy DM, Young JD. A systematic review and meta-analysis of early goal-directed therapy for septic shock: the ARISE, ProCESS and ProMISe Investigators. Intensive Care Med. 2015;41:1549-60.

21. Higgins JPT, Green S, Cochrane Collaboration. Cochrane handbook for systematic reviews of interventions. Chichester: WileyBlackwell; 2008.

22. Moher D, Liberati A, Tetzlaff J, Altman DG. Preferred reporting items for systematic reviews and meta-analyses: the PRISMA statement. PLoS Med. 2009;6:e1000097.

23. Guyatt GH, Oxman AD, Vist GE, Kunz R, Falck-Ytter Y, Alonso-Coello P, Schunemann HJ, Group GW. GRADE: an emerging consensus on rating quality of evidence and strength of recommendations. BMJ 2008;336:924-6.

24. Roberts I, Ker K, Edwards P, Beecher D, Manno D, Sydenham E. The knowledge system underpinning healthcare is not fit for purpose and must change. BMJ (Clin Res Ed). 2015;350:h2463.

25. Wetterslev J, Thorlund K, Brok J, Gluud C. Trial sequential analysis may establish when firm evidence is reached in cumulative meta-analysis. J Clin Epidemiol. 2008;61:64-75.

26. Thorlund K, Engstrøm J, Wetterslev J, Brok J, Imberger G, Gluud C. User manual for trial sequential analysis (TSA). Copenhagen, Denmark: Copenhagen Trial Unit, Centre for Clinical Intervention Research; 2011. p. 1-115. Available from www. ctu.dk/tsa.

27. Kuan WS, Ibrahim I, Leong BS, Jain S, Lu Q, Cheung YB, Mahadevan M. Emergency department management of sepsis patients: a randomized, goal-oriented, noninvasive sepsis trial. Ann Emerg Med. 2016;67(367-378):e363.

28. Holm C, Mayr M, Tegeler J, Horbrand F, Henckel von Donnersmarck G, Muhlbauer W, Pfeiffer UJ. A clinical randomized study on the effects of invasive monitoring on burn shock resuscitation. Burns. 2004;30:798-807.

29. Pearse R, Dawson D, Fawcett J, Rhodes A, Grounds RM, Bennett ED. Early goal-directed therapy after major surgery reduces complications and duration of hospital stay. A randomised, controlled trial [ISRCTN38797445]. Crit Care. 2005;9:R687-93.

30. Chytra I, Pradl R, Bosman R, Pelnar P, Kasal E, Zidkova A. Esophageal Doppler-guided fluid management decreases blood lactate levels in multiple-trauma patients: a randomized controlled trial. Crit Care. 2007;11:R24.

31. Jones AE, Shapiro NI, Trzeciak S, Arnold RC, Claremont HA, Kline JA, Emergency Medicine Shock Research Network, I. Lactate clearance vs central venous oxygen saturation as goals of early sepsis therapy: a randomized clinical trial. JAMA. 2010;303:739-46.

32. Zhang Z, Ni H, Qian Z. Effectiveness of treatment based on PiCCO parameters in critically ill patients with septic shock and/or acute 
respiratory distress syndrome: a randomized controlled trial. Intensive Care Med. 2015;41:444-51.

33. Wheeler AP, Bernard GR, Thompson BT, Schoenfeld D, Wiedemann HP, deBoisblanc B, Connors AF Jr, Hite RD, Harabin AL. Pulmonary-artery versus central venous catheter to guide treatment of acute lung injury. N Engl J Med. 2006;354:2213-24.

34. Jhanji S, Vivian-Smith A, Lucena-Amaro S, Watson D, Hinds CJ, Pearse RM. Haemodynamic optimisation improves tissue microvascular flow and oxygenation after major surgery: a randomised controlled trial. Crit Care. 2010;14:R151.

35. McKendry M, McGloin H, Saberi D, Caudwell L, Brady AR, Singer M. Randomised controlled trial assessing the impact of a nurse delivered, flow monitored protocol for optimisation of circulatory status after cardiac surgery. BMJ (Clin Res Ed). 2004;329:258.

36. Wira CR, Dodge K, Sather J, Dziura J. Meta-analysis of protocolized goal-directed hemodynamic optimization for the management of severe sepsis and septic shock in the Emergency Department. West J Emerg Med. 2014;15:51-9.

37. Barochia AV, Cui X, Vitberg D, Suffredini AF, O'Grady NP, Banks SM, Minneci P, Kern SJ, Danner RL, Natanson C, Eichacker PQ. Bundled care for septic shock: an analysis of clinical trials. Crit Care Med. 2010;38:668-78.

38. Gu WJ, Wang F, Bakker J, Tang L, Liu JC. The effect of goaldirected therapy on mortality in patients with sepsis - earlier is better: a meta-analysis of randomized controlled trials. Crit Care. 2014; 18:570.

39. Tuchschmidt J, Fried J, Astiz M, Rackow E. Elevation of cardiac output and oxygen delivery improves outcome in septic shock. Chest. 1992;102:216-20.

40. Yu M, Levy MM, Smith P, Takiguchi SA, Miyasaki A, Myers SA. Effect of maximizing oxygen delivery on morbidity and mortality rates in critically ill patients: a prospective, randomized, controlled study. Crit Care Med. 1993;21:830-8.

41. Yu M, Burchell S, Hasaniya NW, Takanishi DM, Myers SA, Takiguchi SA. Relationship of mortality to increasing oxygen delivery in patients $>$ or $=50$ years of age: a prospective, randomized trial. Crit Care Med. 1998;26:1011-9.

42. Gattinoni L, Brazzi L, Pelosi P, Latini R, Tognoni G, Pesenti A, Fumagalli R. A trial of goal-oriented hemodynamic therapy in critically ill patients. SvO2 Collaborative Group. N Engl J Med. 1995;333:1025-32.

43. Alia I, Esteban A, Gordo F, Lorente JA, Diaz C, Rodriguez JA, Frutos F. A randomized and controlled trial of the effect of treatment aimed at maximizing oxygen delivery in patients with severe sepsis or septic shock. Chest. 1999;115:453-61.

44. Peake SL, Bailey M, Bellomo R, Cameron PA, Cross A, Delaney A, Finfer S, Higgins A, Jones DA, Myburgh JA, Syres GA, Webb SA, Williams P. Australasian resuscitation of sepsis evaluation (ARISE): A multi-centre, prospective, inception cohort study. Resuscitation. 2009;80:811-8.

45. de Oliveira CF, de Oliveira DS, Gottschald AF, Moura JD, Costa GA, Ventura AC, Fernandes JC, Vaz FA, Carcillo JA, Rivers EP, Troster EJ. ACCM/PALS haemodynamic support guidelines for paediatric septic shock: an outcomes comparison with and without monitoring central venous oxygen saturation. Intensive Care Med. 2008;34:1065-75.

46. Wang XZ, Lu CJ, Gao FQ, Li XH, Yan WF, Ning FY. Efficacy of goal-directed therapy in the treatment of septic shock. Zhongguo Wei Zhong Bing Ji Jiu Yi Xue 2006;18:661-664.

47. Province EG-DTCGoZ. The effect of early goal-directed therapy on treatment of critical patients with severe sepsis/septic shock: a multi-center, prospective, randomized, controlled study. Zhongguo Wei Zhong Bing Ji Jiu Yi Xue. 2010;22:331-334.

48. Tian HH, Han SS, Lv CJ, Wang T, Li Z, Hao D, Shang QM, Wang $\mathrm{XZ}$. The effect of early goal lactate clearance rate on the outcome of septic shock patients with severe pneumonia. Zhongguo Wei Zhong Bing Ji Jiu Yi Xue. 2012;24:42-45.

49. Yu B, Tian HY, Hu ZJ, Zhao C, Liu LX, Zhang Y, Zhu GJ, Wang LT, Wu XH, Li J. Comparison of the effect of fluid resuscitation as guided either by lactate clearance rate or by central venous oxygen saturation in patients with sepsis. Zhonghua Wei Zhong Bing Ji Jiu Yi Xue. 2013;25:578-83.

50. Lu N, Zheng R, Lin H, Shao J, Yu J. Clinical studies of surviving sepsis bundles according to PiCCO on septic shock patients. Zhonghua Wei Zhong Bing Ji Jiu Yi Xue. 2014;26:23-7.

51. Teboul JL, Saugel B, Cecconi M, De Backer D, Hofer CK, Monnet X, Perel A, Pinsky MR, Reuter DA, Rhodes A, Squara P, Vincent JL, Scheeren TW. Less invasive hemodynamic monitoring in critically ill patients. Intensive Care Med. 2016;42:1350-9.

52. Critchley LA, Critchley JA. A meta-analysis of studies using bias and precision statistics to compare cardiac output measurement techniques. J Clin Monit Comput. 1999;15:85-91.

53. Critchley LA. Bias and precision statistics: should we still adhere to the $30 \%$ benchmark for cardiac output monitor validation studies? Anesthesiology 2011;114:1245-1246.

54. Hapfelmeier A, Cecconi M, Saugel B. Cardiac output method comparison studies: the relation of the precision of agreement and the precision of method. J Clin Monit Comput. 2016;30:149-55.

55. National Heart L, Blood Institute Acute Respiratory Distress Syndrome Clinical Trials N, Wheeler AP, Bernard GR, Thompson BT, Schoenfeld D, Wiedemann HP, deBoisblanc B, Connors AF $\mathrm{Jr}$, Hite RD, Harabin AL. Pulmonary-artery versus central venous catheter to guide treatment of acute lung injury. N Engl J Med. 2006;354:2213-2224.

56. Goedje O, Hoeke K, Lichtwarck-Aschoff M, Faltchauser A, Lamm $\mathrm{P}$, Reichart B. Continuous cardiac output by femoral arterial thermodilution calibrated pulse contour analysis: comparison with pulmonary arterial thermodilution. Crit Care Med. 1999;27:2407-12.

57. Mora B, Ince I, Birkenberg B, Skhirtladze K, Pernicka E, Ankersmit HJ, Dworschak M. Validation of cardiac output measurement with the LiDCO pulse contour system in patients with impaired left ventricular function after cardiac surgery. Anaesthesia. 2011;66:675-81.

58. Molnar Z, Umgelter A, Toth I, Livingstone D, Weyland A, Sakka SG, Meier-Hellmann A. Continuous monitoring of $\mathrm{ScvO}(2)$ by a new fibre-optic technology compared with blood gas oximetry in critically ill patients: a multicentre study. Intensive Care Med. 2007;33:1767-70.

59. Peyton PJ, Chong SW. Minimally invasive measurement of cardiac output during surgery and critical care: a meta-analysis of accuracy and precision. Anesthesiology. 2010;113:1220-35.

60. Squara P, Denjean D, Estagnasie P, Brusset A, Dib JC, Dubois C. Noninvasive cardiac output monitoring (NICOM): a clinical validation. Intensive Care Med. 2007;33:1191-4.

61. Stawicki PS, Braslow B, Gracias VH. Exploring measurement biases associated with esophageal Doppler monitoring in critically ill patients in intensive care unit. Ann of Thorac Med. 2007;2:148-53.

62. Cecconi M, De Backer D, Antonelli M, Beale R, Bakker J, Hofer C, Jaeschke R, Mebazaa A, Pinsky MR, Teboul JL, Vincent JL, Rhodes A. Consensus on circulatory shock and hemodynamic monitoring. Task force of the European Society of Intensive Care Medicine. Intensive Care Med. 2014;40:1795-815.

63. van Beest PA, Hofstra JJ, Schultz MJ, Boerma EC, Spronk PE, Kuiper MA. The incidence of low venous oxygen saturation on admission to the intensive care unit: a multi-center observational study in The Netherlands. Crit Care. 2008;12:R33.

64. Squara P. Central venous oxygenation: when physiology explains apparent discrepancies. Crit Care. 2014;18:579.

65. Saugel B, Malbrain ML, Perel A. Hemodynamic monitoring in the era of evidence-based medicine. Crit Care. 2016;20:401. 